\title{
Conceptual design and kinematic analysis of a novel parallel robot for high-speed pick-and-place operations
}

(C) The Author(s) 2017. This article is published with open access at link.springer.com and journal.hep.com.cn

\begin{abstract}
This paper deals with the conceptual design, kinematic analysis and workspace identification of a novel four degrees-of-freedom (DOFs) high-speed spatial parallel robot for pick-and-place operations. The proposed spatial parallel robot consists of a base, four arms and a $1 \frac{1}{2}$ mobile platform. The mobile platform is a major innovation that avoids output singularity and offers the advantages of both single and double platforms. To investigate the characteristics of the robot's DOFs, a line graph method based on Grassmann line geometry is adopted in mobility analysis. In addition, the inverse kinematics is derived, and the constraint conditions to identify the correct solution are also provided. On the basis of the proposed concept, the workspace of the robot is identified using a set of presupposed parameters by taking input and output transmission index as the performance evaluation criteria.
\end{abstract}

Keywords spatial parallel robot, pick-and-place operations, mobility analysis, kinematic modeling, workspace identification

\section{Introduction}

As indispensable equipment in advanced manufacturing systems, industrial robots have become an important symbol to measure the level of a country's manufacturing industry. Due to the increasing demand for cost efficiency,

Received January 29, 2017; accepted June 13, 2017

Qizhi MENG, Fugui XIE ( $₫)$, Xin-Jun LIU ( $₫)$

The State Key Laboratory of Tribology \& Institute of Manufacturing Engineering, Department of Mechanical Engineering, Tsinghua University, Beijing 100084, China

E-mail: xiefg@mail.tsinghua.edu.cn; xinjunliu@mail.tsinghua.edu.cn

Fugui XIE, Xin-Jun LIU

Beijing Key Laboratory of Precision/Ultra-precision Manufacturing Equipments and Control, Tsinghua University, Beijing 100084, China such robots have been widely used to implement highspeed manipulations of sorting and packaging in the food, pharmaceuticals, and electronics industries. As the most representative and most significant application category, pick-and-place operations have received extensive research attention. In fact, for most pick-and-place operations, at least four degrees-of-freedom (DOFs) are required, including three translations (3T) to move the object from one point to another and one rotation (1R) to adjust the posture in its final location [1]. This kind of DOFs is usually called SCARA motion [2-4] or Schönflies motion [5-7]. The motions are generally characterized by high flexibility and stiffness respectively in the horizontal and vertical directions.

In the production lines, the operational objectives are generally in small size and light weight. The first robot for pick-and-place operations was realized by serial kinematic mechanisms (SKMs), whose drive units were placed within the joints. This placement leads to a large volume and high inertia force. On the contrary, parallel kinematic mechanisms (PKMs) are advantageous over serial structures due to their high rigidity, high precision and better dynamic characteristics $[8,9]$. In relation to this, the most famous Delta robot $[10,11]$ with only $3 \mathrm{~T}$ for parallel architecture has been proposed by Clavel [12]. This Delta robot, a milestone for high-speed parallel robots, has become a real commercial success with a large workspace and compact structure. Based on the configuration of Delta, a passive RUPUR chain has been adopted to realize the rotary output. This scheme has a wide range of applications, such as the ABB IRB340 FlexPicker robot [13] and the Bosch SIGPack Systems XR22 robot. However, the serially added device may become a weak point and may lead to the lack of stiffness and short service life [14-16]. To avoid this disadvantage, Pierrot et al. [1,17-20] proposed a new family of 4-DOF, doubleplatform parallel robots called $\mathrm{H} 4$. The rotary output of $\mathrm{H} 4$ is realized by the relative movement of the two platforms. Notably, the double-platform structure may lead to 
structural complexity and difficulty in manufacturing. Therefore, in this work, we present a parallel robot with concise and compact structure as well as good performance.

As shown in Fig. 1, the double-platform structure improved from H4, which has been adopted by Par4 [16], demonstrates good rotational performance; however, it also leads to a complex structure, increased difficulty in manufacturing, and higher accessory cost. Meanwhile, the single platform adopted by X4 [21,22], contributes a simple structure and no amplification mechanism, but has worse performance when the platform is close to the maximum output angle. Therefore, developing a $1 \frac{1}{2}$ mobile platform that carries the merits of both single and double platforms provides an entry point for new parallel robots. The design and development of a high-speed parallel robot with simple structure and high performance is still a hot issue in both the academic and industrial fields.

The remainder of this paper is organized into sections. The conceptual design of the proposed parallel robot is introduced in Section 2. The mobility analysis is carried out in Section 3. The inverse kinematics is investigated in Section 4, and the workspaces are identified in Section 5. Finally, Section 6 concludes the paper.

\section{Conceptual design}

The architecture of the proposed parallel robot, shown in Fig. 2(a), consists of a base (Part 1), four limbs (I, II, III, and IV), and a $1 \frac{1}{2}$ mobile platform (Assembly 8 ) with an end effector. The four limbs are connected to the base and mobile platform, and collectively, these components make up the spatial closed-loop mechanism. As presented in Fig. 2(b), the layouts of the four arms are symmetrically arranged in a circular manner.

The four limbs have two kinds of structures and can be summarized as follows. Limbs I and IV have the same structure, represented by $\mathrm{R}-\mathrm{R}-(\mathrm{Pa})-\mathrm{R}$, where $\mathrm{R}$ represents an active revolute joint, $\mathrm{R}$ indicates the revolute joint, and Pa denotes the planar parallelogram mechanism consisting of four revolute joints connected end to end. Compared with Limbs I and IV, Limbs II and III have an additional R and can be expressed as R-R-(Pa)-R-R. For better understanding, the joint-and-loop graph for the basic principle is illustrated in Fig. 3, where the lines and boxes represent the parts and the joints, respectively.

Figure 4(a) shows the structure of the $1 \frac{1}{2}$ mobile platform, which consists of an upper platform, a lower

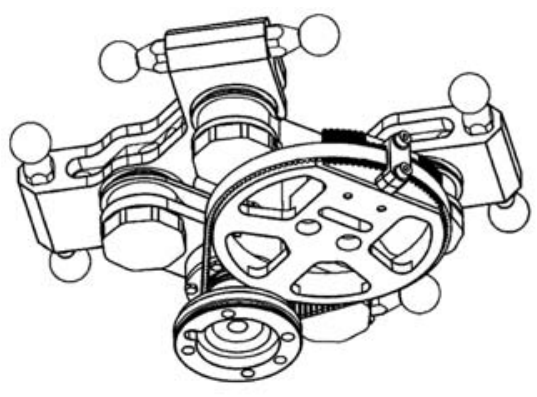

(a)

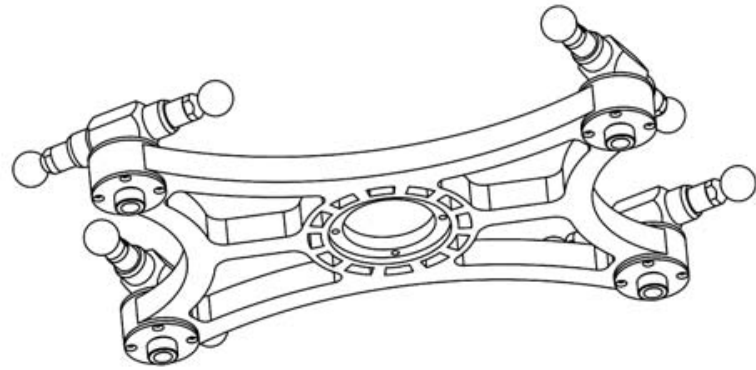

(b)

Fig. 1 CAD models of different mobile platforms. (a) Double-platform structure of H4; (b) single-platform structure of X4

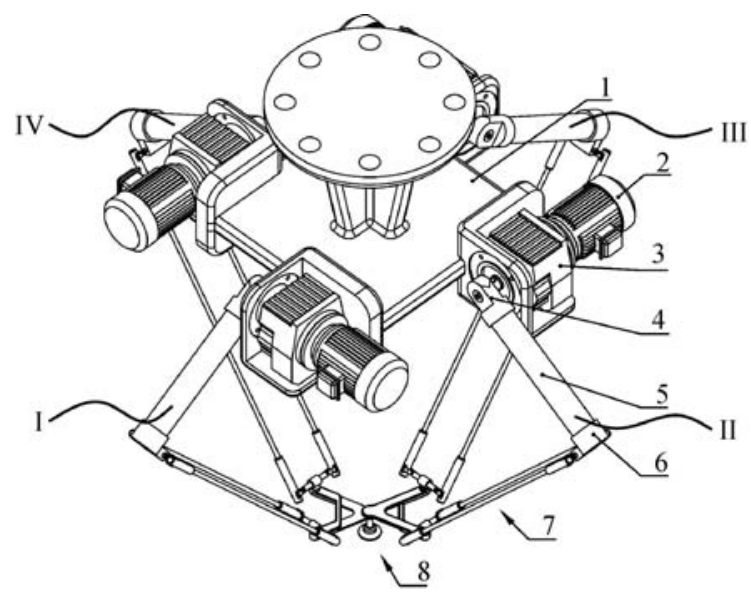

(a)

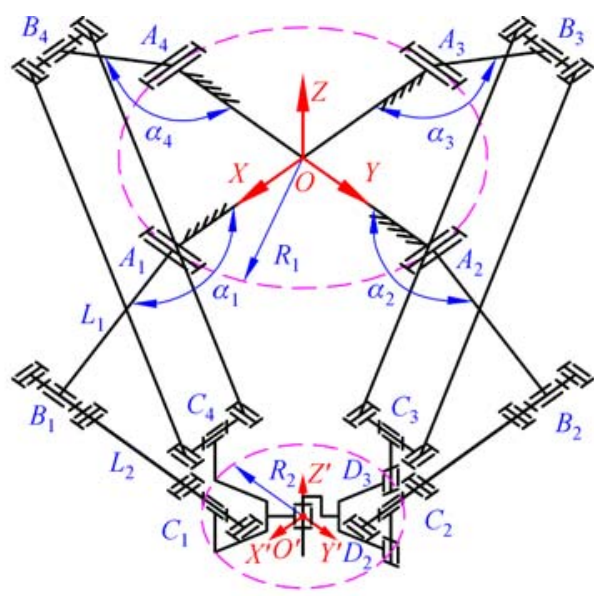

(b)

Fig. 2 Architecture of the proposed parallel robot. (a) Conceptual model; (b) kinematic model 


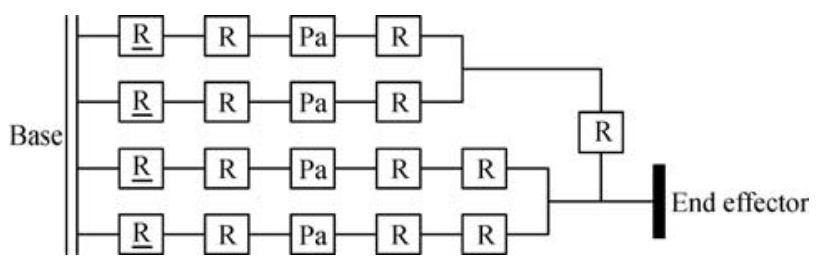

Fig. 3 The joint-and-loop graph for basic principle

platform, and an end effector; the upper and lower platforms are connected by a revolute Joint R. The meaning of the " $1 \frac{1}{2}$ mobile platform" comes from the total contributions of platforms to output motions. As an auxiliary output platform, the lower platform generates incomplete output motions (3T). The upper platform providing the complete output motions (3T1R) is defined as the final output platform; therefore, they are treated as $1 / 2$ and 1 mobile platforms, respectively, and together constitute a generalized mobile platform.

An equivalent kinematic model of the proposed parallel robot is developed, as shown in Fig. 2(b). The active and passive arms in each limb are represented by $A_{i} B_{i}$ and $B_{i} C_{i}$, respectively. The junction points between the base and active arms are denoted as $A_{i}$, whereas the central points of the passive revolute joints between the active and passive arms are denoted as $B_{i}$. The connective points between the passive arms and mobile platform are denoted as $C_{i}$. A fixed global reference frame $\mathfrak{R}: O-X Y Z$ is located at the central point of the base. The $X$ - and $Z$-axes are coincident with $A_{3} A_{1}$ and $A_{4} A_{2}$, respectively. Another reference frame, called the moving frame $\Re^{\prime}: O^{\prime}-X^{\prime} Y^{\prime} Z^{\prime}$, is located at the central point of the lower platform. The kinematic

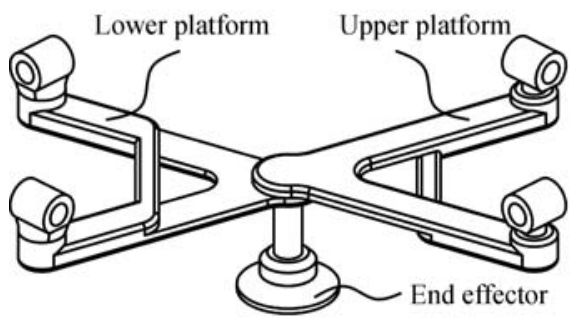

(a) model in the initial position of the mobile platform is shown in Fig. 4(b), where $C_{1} C_{2} C_{3} C_{4}$ is considered as a rectangle (i.e., $\angle D_{2} O^{\prime} D_{3} \neq 90^{\circ}$ ) to avoid singularity.

\section{Mobility analysis}

This section considers a line-graph method $[23,24]$ based on Grassmann line geometry $[25,26]$ to analyze the mobility of the presented spatial parallel mechanism. The principle for the articulated mobile platform to generate the 3T1R motions are described in detail.

\subsection{Line-graph method}

According to Grassmann line geometry, spatial line clusters can be divided into five types from 1 to 5 , corresponding to their respective dimensions. In addition, an N-dimensional spatial line cluster, which consists of vector or couple in two different colors, represents a freedom space or constraint space for a mechanism. To depict the spatial line cluster, four basic elements with different mathematic and physical meanings are generated in Table 1. The manner of adopting line graphs to describe mobility is generally referred to as line-graph method.

\subsection{Basic rules}

We can extract the motion line graphs of each limb from the architecture of the presented mechanism. Unlike the traditional SKM, the motions of the end effector of PKM cannot be obtained by the simple superposition of the

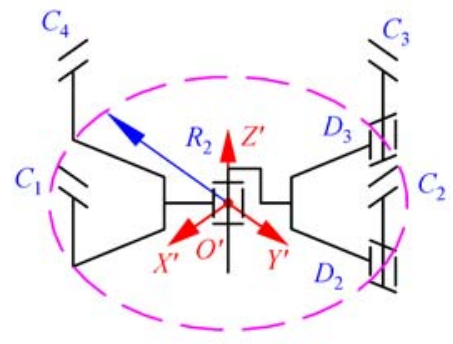

(b)

Fig. 4 The architecture of the mobile platform. (a) Conceptual model, (b) kinematic model

Table 1 Graphic expressions of the basic elements and their meanings

\begin{tabular}{lccc}
\hline Graphic expressions & Mathematical meanings & Physical meanings & Screw representation \\
\hline & Vector & Rotational motion & $(\boldsymbol{\omega} ; \boldsymbol{r} \times \boldsymbol{\omega})$ \\
& Vector & Constraint force & $(\boldsymbol{f} ; \boldsymbol{r} \times \boldsymbol{f})$ \\
& Couple & Translational motion & $(0 ; \boldsymbol{v})$ \\
\hline
\end{tabular}


motions of each limb. In other words, the motion line graphs of the mobile platform cannot be directly accessed from the motion line graphs of the branched chains.

Furthermore, the branched chain provides all the necessary constraints for the mobile platform, and these constraints are superimposed. Therefore, finding a way to establish an equivalent conversion between motion and constraint line graphs is quite indispensable. Based on the above analysis, a generalized Blanding rule [27], which states that there is no power between the constraint forces and the allowable motions as a foundation, is introduced and illustrated in Fig. 5. This is further summarized below.

Basic rule A. Every vector of the motion/constraint line graphs intersects with all vectors of the corresponding constraint/motion line graphs (in projective theory, parallel lines are seen as a special intersection). Hence, $(\boldsymbol{\omega} ; \boldsymbol{r} \times \boldsymbol{\omega})^{\circ}$ $(\boldsymbol{f} ; \boldsymbol{r} \times \boldsymbol{f})=0$, but only when $\boldsymbol{f}$ and $\boldsymbol{\omega}$ intersect at one point, where the symbol $\circ$ represents reciprocal product between two screws in the screw theory.

Basic rule B. Every vector of the motion/constraint line graphs is orthogonal to all couples of the corresponding constraint/motion line graphs. Hence, $(\boldsymbol{f} ; \boldsymbol{r} \times \boldsymbol{f})^{\circ}(\mathbf{0} ; \boldsymbol{v})=0$

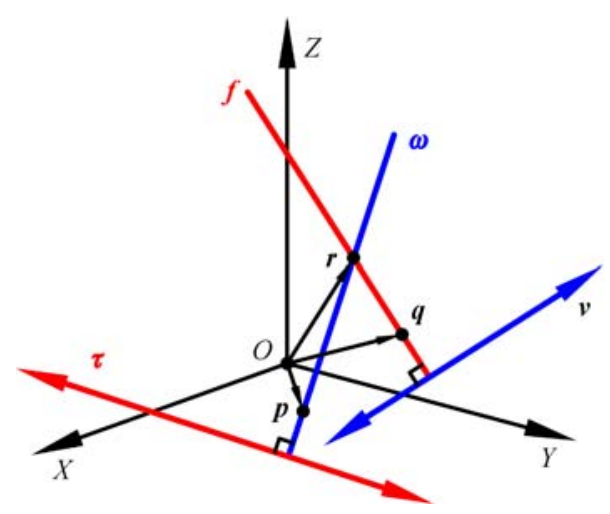

Fig. 5 Graphical representations of the basic rules

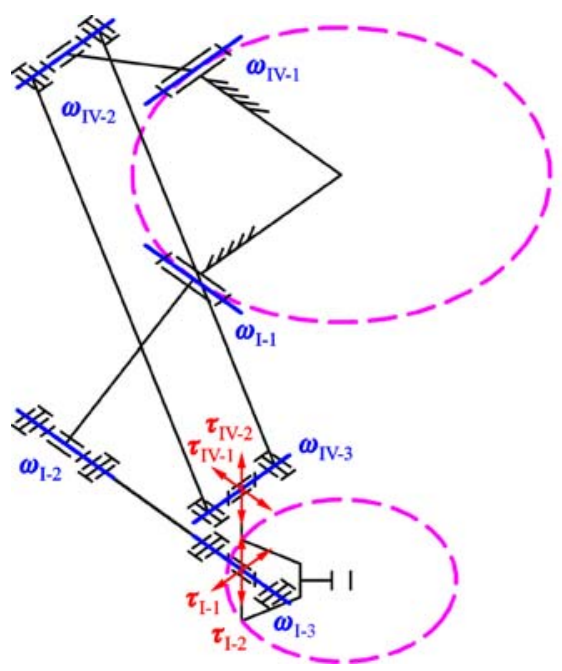

or $(0 ; \boldsymbol{\tau})^{\circ}(\boldsymbol{\omega} ; \boldsymbol{r} \times \boldsymbol{\omega})=0$, but only when $\boldsymbol{f}$ and $\boldsymbol{v}$ or $\boldsymbol{\tau}$ and $\boldsymbol{\omega}$ are perpendicular to each other.

Basic rule C. The directions between the couple of motion/constraint line graphs and the corresponding constraint/motion line graphs are arbitrary. Obviously, (0; $\boldsymbol{\tau})^{\circ}(\mathbf{0} ; \boldsymbol{v})=0$.

\subsection{Analysis results}

Four steps are necessary to investigate the mobility of the mechanism. First, the motion line graph of each limb is extracted. Second, the corresponding constraint line graph of each limb are derived using the basic rules. Third, all the constraint line graphs of the four limbs to the mobile platform are added. Finally, the corresponding motion line graph of the mobile platform are derived.

Four limbs can be classified into two categories according to their structural characteristics. One group embraces Limbs I and IV, and the other group includes Limbs II and III. The mobility analysis of the proposed mechanism can be carried out based on the four steps described below.

1) Limb I is taken as a case study because of the same configuration of Limbs I and IV. As shown in Fig. 6(a), the motion line graphs consist of three rotations $\left(\omega_{\mathrm{I}-1}, \omega_{\mathrm{I}-2}, \omega_{\mathrm{I}-3}\right)$ and a translation $\left(\boldsymbol{v}_{\mathrm{I}-1}\right)$ located in the plane of the parallelogram and orthogonal to $B_{1} C_{1}$. Therefore, the motion line graph has four dimensions, and the corresponding constraint line graph must be 2D. According to basic rule $\mathrm{B}$, every couple in the constraint line graph should be orthogonal to all the vectors $\left(\omega_{\mathrm{I}-1}, \omega_{\mathrm{I}-2}, \omega_{\mathrm{I}-3}\right)$ of the motion line graph. In this way, $\tau_{\mathrm{I}-1}$ and $\boldsymbol{\tau}_{\mathrm{I}-2}$, which lie at Point $C_{1}$ and are parallel to the $X$ - and $Z$-axes, respectively, can be derived. According to basic rules $\mathrm{A}$ and $\mathrm{B}$, a vector in the constraint line graph should intersect all the vectors $\left(\omega_{\mathrm{I}-1}, \omega_{\mathrm{I}-2}, \omega_{\mathrm{I}-3}\right)$ and must be orthogonal to all the couples $\left(v_{\mathrm{I}-1}\right)$ of the motion line graph; hence, no vector $\left(f_{\mathrm{I}-1}\right)$ can

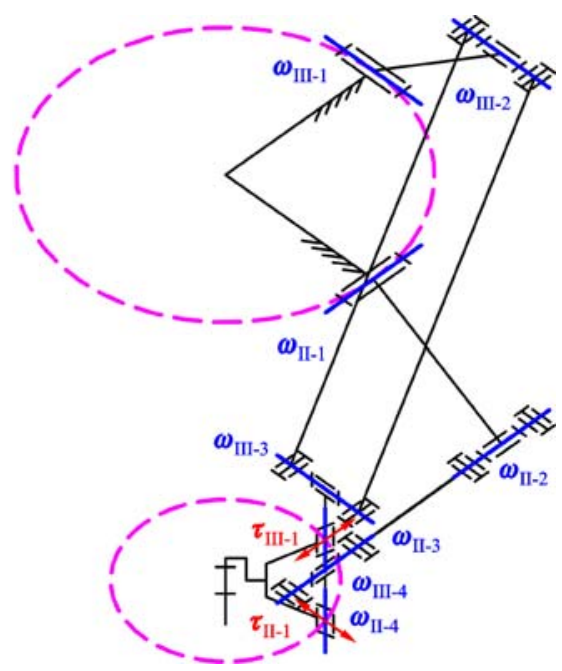

Fig. 6 Motion and constraint line graphs for two groups of limbs. (a) Limbs I and IV; (b) Limbs II and III 
be found. Together, the constraint line graph of Limb I is a 2D couple constraint $\left(\boldsymbol{\tau}_{\mathrm{I}-1}\right.$ and $\left.\boldsymbol{\tau}_{\mathrm{I}-2}\right)$.

2) Limb II is taken as a case study because of the same configuration of Limbs II and III. As shown in Fig. 6(b), the motion line graph consists of four rotations $\left(\omega_{\mathrm{II}-1}, \omega_{\mathrm{II}-2}, \omega_{\mathrm{II}-3}, \omega_{\mathrm{II}-4}\right)$ located at Points $A_{2}, B_{2}, C_{2}$, and $D_{2}$, respectively, and a translation $\left(v_{\mathrm{II}-1}\right)$ lying on the plane of the parallelogram and is orthogonal to $B_{2} C_{2}$. Therefore, the motion line graph has five dimensions, and the corresponding constraint line graph must be $1 \mathrm{D}$. According to basic rule $\mathrm{B}$, every couple in the constraint line graph should be orthogonal to all the vectors $\left(\omega_{\mathrm{II}-1}, \omega_{\mathrm{II}-2}, \omega_{\mathrm{II}-3}, \omega_{\mathrm{II}-4}\right)$ of the motion line graph, and $\boldsymbol{\tau}_{\mathrm{II}-1}$ must lie at Point $D_{2}$ and is parallel to the $Y$-axis. According to basic rules A and $\mathrm{B}$, a vector in the constraint line graph should be intersected to all the vectors $\left(\omega_{\mathrm{II}-1}, \omega_{\mathrm{II}-2}, \omega_{\mathrm{II}-3}, \omega_{\mathrm{II}-4}\right)$ and orthogonal to all the couples $\left(v_{\mathrm{II}-1}\right)$ of the motion line graph; hence, no vector $\left(f_{\text {II-1 }}\right)$ can be found. Together, the constraint line graph of Limb II is a $1 \mathrm{D}$ couple constraint $\left(\tau_{\mathrm{II}-1}\right)$.

3) For the $1 \frac{1}{2}$ mobile platform, on the one hand, the constraints imposed on the lower platform can be obtained through the superposition of the constraints of Limb I ( $\tau_{\mathrm{I}-1}$ and $\left.\boldsymbol{\tau}_{\mathrm{I}-2}\right)$, Limb IV $\left(\boldsymbol{\tau}_{\mathrm{IV}-1}\right.$ and $\left.\boldsymbol{\tau}_{\mathrm{IV}-2}\right)$, and the upper platform $\left(\boldsymbol{\tau}_{\mathrm{U}-1}\right.$ and $\boldsymbol{\tau}_{\mathrm{U}-2}$, which resulted from $\boldsymbol{\tau}_{\mathrm{II}-1}$ and $\boldsymbol{\tau}_{\mathrm{III}-3}$ provided by Limbs II and III). The constraint line graph of the lower platform can be derived by processes (1), (2), and (3) shown in Fig. 7. The six couple constraints $\left(\boldsymbol{\tau}_{\mathrm{I}-1}, \boldsymbol{\tau}_{\mathrm{I}-2}, \boldsymbol{\tau}_{\mathrm{IV}-1}\right.$, $\left.\boldsymbol{\tau}_{\mathrm{IV}-2}, \boldsymbol{\tau}_{\mathrm{U}-1}, \boldsymbol{\tau}_{\mathrm{U}-2}\right)$ together construct a $3 \mathrm{D}$ couple constraint (equivalent to $\boldsymbol{\tau}_{\mathrm{L}-1}, \boldsymbol{\tau}_{\mathrm{L}-2}$, and $\boldsymbol{\tau}_{\mathrm{L}-3}$ ). Therefore, three DOFs with $3 \mathrm{~T}$ motions of the lower platform are identified. On the other hand, the constraints imposed on the upper platform can be obtained through the superposition of the constraints of Limb II $\left(\boldsymbol{\tau}_{\text {II-1 }}\right)$, Limb III $\left(\boldsymbol{\tau}_{\text {III-1 }}\right)$, and the lower platform $\left(\boldsymbol{\tau}_{\mathrm{L}-1}, \boldsymbol{\tau}_{\mathrm{L}-2}\right.$, and $\boldsymbol{\tau}_{\mathrm{L}-3}$, which resulted from $\boldsymbol{\tau}_{\mathrm{I}-1}, \boldsymbol{\tau}_{\mathrm{I}-2}$, $\boldsymbol{\tau}_{\mathrm{IV}-1}$ and $\boldsymbol{\tau}_{\mathrm{IV}-2}$ provided by Limbs I and IV). The constraint line graph of the upper platform can be derived by processes (2), (1), and (4) shown in Fig. 7. Because of the revolute joint between the lower and upper platforms, the five couple constraints $\left(\boldsymbol{\tau}_{\mathrm{II}-1}, \boldsymbol{\tau}_{\mathrm{III}-1}, \boldsymbol{\tau}_{\mathrm{L}-1}, \boldsymbol{\tau}_{\mathrm{L}-2}, \boldsymbol{\tau}_{\mathrm{L}-3}\right)$ together construct a $2 \mathrm{D}$ couple constraint (equivalent to $\boldsymbol{\tau}_{\mathrm{U}-1}$ and
$\left.\boldsymbol{\tau}_{\mathrm{U}-2}\right)$. Therefore, four DOFs with $3 \mathrm{~T}$ and $1 \mathrm{R}$ motions of the upper platform are clarified.

Above all, the output motion spaces of the presented mechanism are $3 \mathrm{~T}$ and $1 \mathrm{R}$, which can be represented by a 4D atlas. In addition, the constraint and motion spaces of different robot configurations are summarized in Table 2. For better understanding, the CAD model with $3 \mathrm{~T}$ and $1 \mathrm{R}$ motions is shown in Fig. 8.

\section{Inverse kinematic investigation}

The geometric parameters of the PKM presented in Fig. 2 are defined as follows: $O A_{i}=R_{1}, O^{\prime} C_{i}=R_{2}, A_{i} B_{i}=L_{1}$, $B_{i} C_{i}=L_{2}$. The distance between $o^{\prime}$ and $C_{2}\left(C_{4}\right)$ along the $Z^{\prime}$-axis direction is denoted by $L_{3}$. The rotational input of the four arms and output of the mobile platform are represented by $\alpha_{i}(i=1,2,3,4)$ and $\theta$, respectively.

As shown in Fig. 2(b), the coordinates of $\boldsymbol{A}_{i}$ $(i=1,2,3,4)$ under the global frame $\Re$ can be respectively expressed by

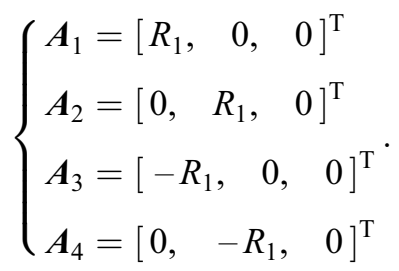

The coordinates of $\boldsymbol{B}_{i}(i=1,2,3,4)$ can be respectively derived as

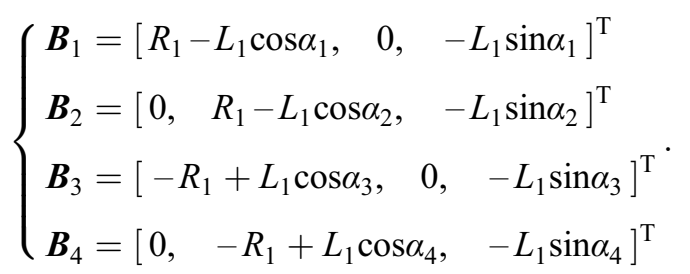

Under the local frame $\mathfrak{R}^{\prime}$, the coordinates of $\boldsymbol{C}_{i}^{\prime}$ $(i=1,2,3,4)$ can be respectively expressed by

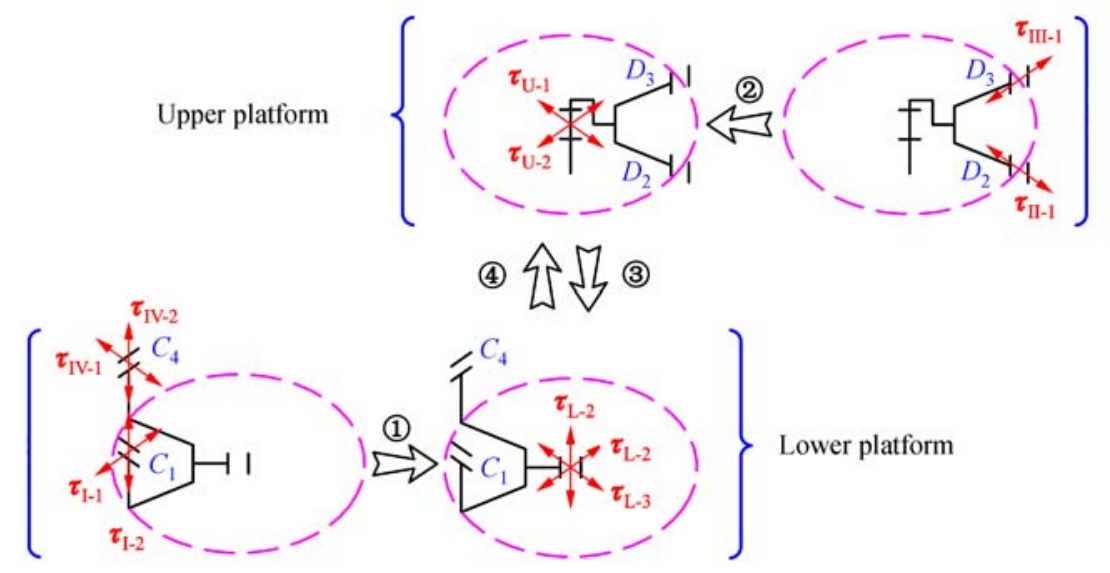

Fig. 7 Constraint line graphs for the lower and upper platforms 
Table 2 Constraint and motion spaces of different configurations to the robot

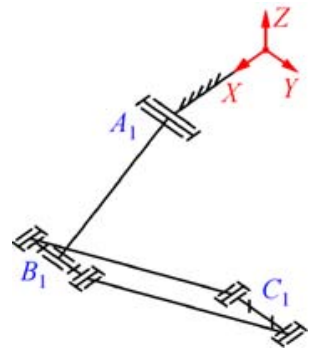

Limb I

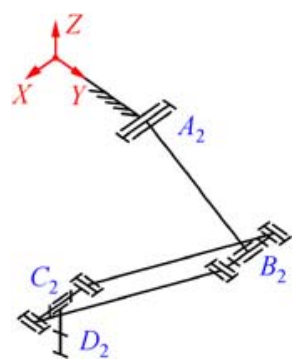

Limb II

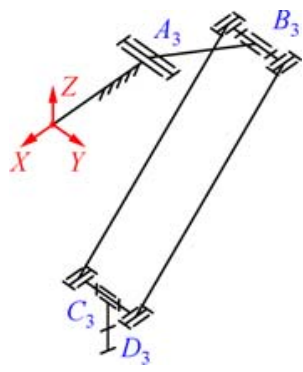

Limb III

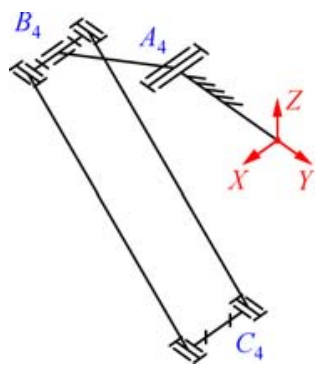

Limb IV

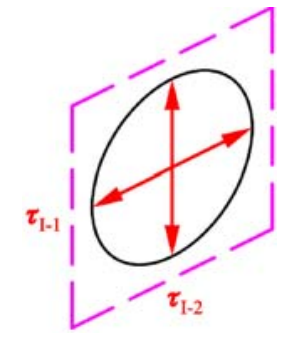

2D couple constraints

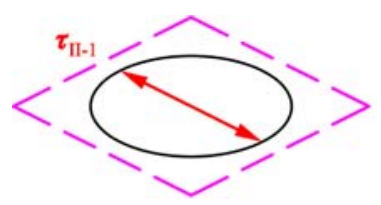

1D couple constraint

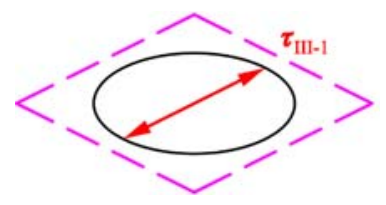

1D couple constraint

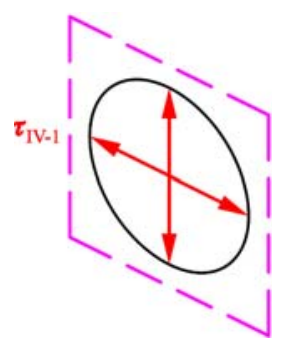

1D rotation and 3D translations
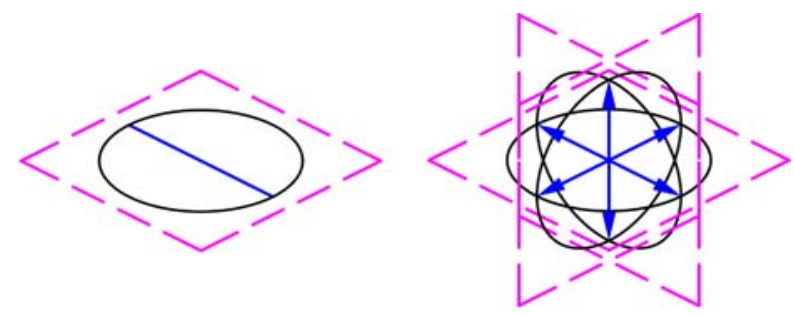

$1 \mathrm{D}$ rotation and $3 \mathrm{D}$ translations
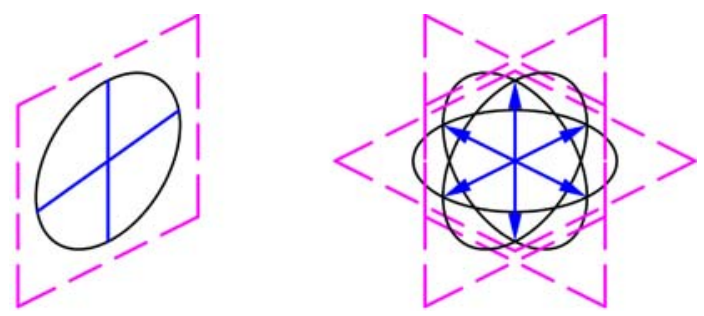

$2 \mathrm{D}$ rotations and $3 \mathrm{D}$ translations
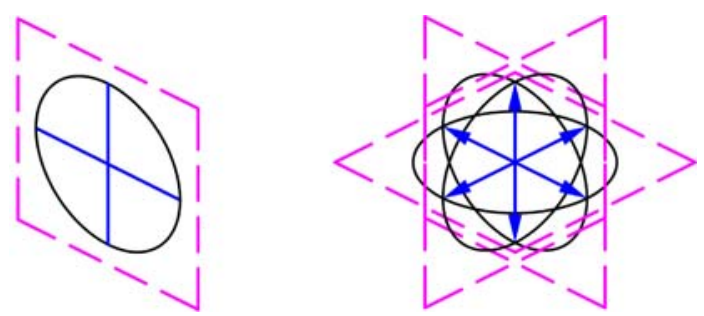

2D rotations and 3D translations
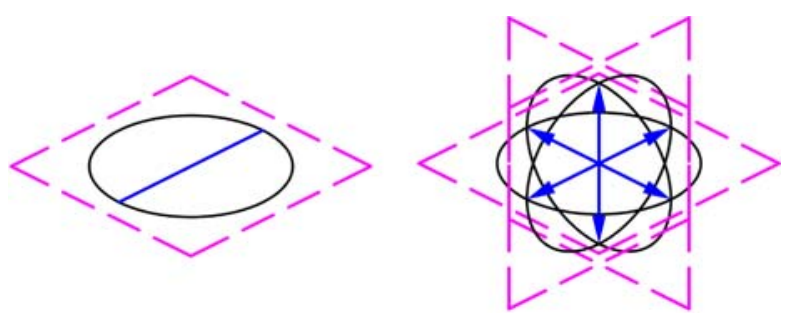

2D couple constraints 


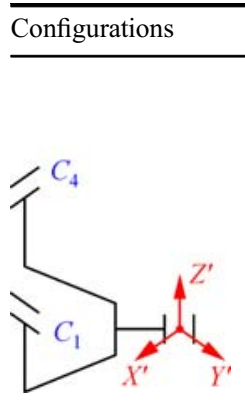

Lower platform

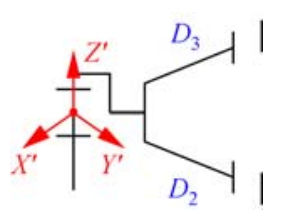

Upper platform

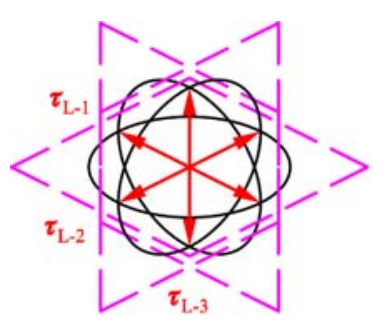

3D couple constraints

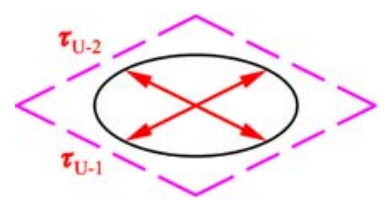

2D couple constraints

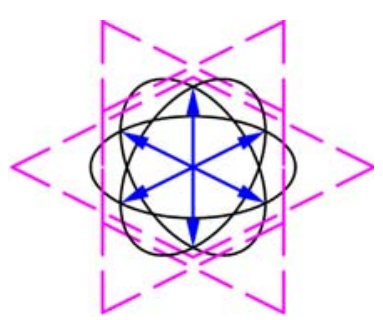

3D translations
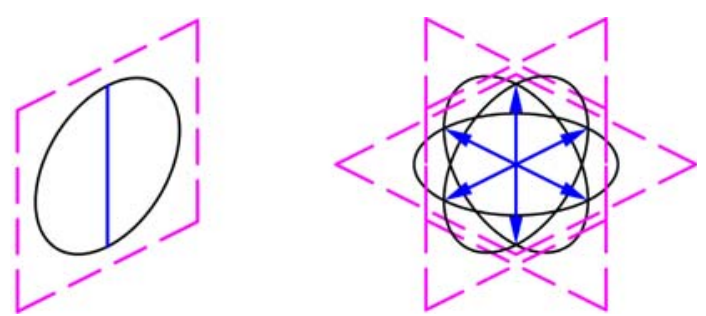

1D rotation and 3D translations

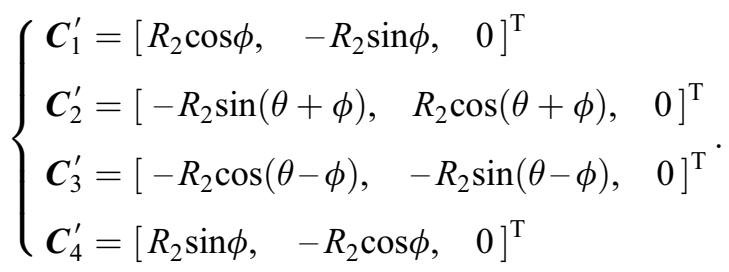

In the coordinates above, $\phi=\frac{90^{\circ}-\left(180^{\circ}-\angle D_{2} O^{\prime} D_{3}\right)}{2}$ $=15^{\circ}$, and the displacement matrix can be expressed as

$$
\boldsymbol{T}=\left[\begin{array}{lll}
x, & y, & z
\end{array}\right]^{\mathrm{T}} .
$$

Based on the vector equation, we arrive at

$$
\boldsymbol{C}_{i}=\boldsymbol{C}_{i}^{\prime}+\boldsymbol{T}
$$

where the coordinates of $\boldsymbol{C}_{i}^{\prime}(i=1,2,3,4)$ under the global frame $\mathfrak{R}$ can be respectively derived as

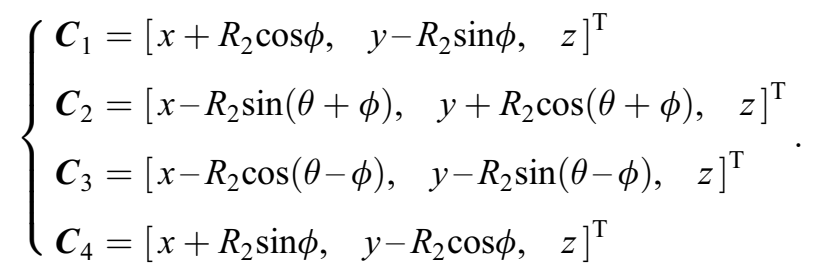

Considering the constraints of $B_{i} C_{i}=L_{2}(i=1,2,3,4)$, the inverse kinematics can be obtained according to Eqs. (2) and (6).

1) The inverse kinematic solution of Limb I is given by

$$
\begin{gathered}
\alpha_{\mathrm{I}-1}=\cos ^{-1} v_{\mathrm{I}}^{+} \text {or } \alpha_{\mathrm{I}-2}=2 \pi-\cos ^{-1} v_{\mathrm{I}}^{+} \\
\text {or } \alpha_{\mathrm{I}-3}=\cos ^{-1} v_{\mathrm{I}}^{-} \text {or } \alpha_{\mathrm{I}-4}=2 \pi-\cos ^{-1} v_{\mathrm{I}}^{-},
\end{gathered}
$$

where

$$
\begin{gathered}
v_{\mathrm{I}}^{ \pm}=\frac{-p_{11} p_{12} \pm \sqrt{4 L_{1}^{2} z_{1}^{2}\left(z_{1}^{2}+p_{12}^{2}\right)-p_{11}^{2} z_{1}^{2}}}{2 L_{1}\left(z_{1}^{2}+p_{12}^{2}\right)} \\
p_{11}=L_{2}^{2}-L_{1}^{2}-\left(R_{1}-x_{1}\right)^{2}-y_{1}^{2}-z_{1}^{2}, \\
p_{12}=R_{1}-x_{1} .
\end{gathered}
$$

2) The inverse kinematic solution of Limb II is expressed as

$$
\begin{gathered}
\alpha_{\mathrm{II}-1}=\cos ^{-1} v_{\mathrm{II}}^{+} \text {or } \alpha_{\mathrm{II}-2}=2 \pi-\cos ^{-1} v_{\mathrm{II}}^{+} \\
\text {or } \alpha_{\mathrm{II}-3}=\cos ^{-1} v_{\mathrm{II}}^{-} \text {or } \alpha_{\mathrm{II}-4}=2 \pi-\cos ^{-1} v_{\mathrm{II}}^{-},
\end{gathered}
$$

where

$$
v_{\mathrm{II}}^{ \pm}=\frac{-p_{21} p_{22} \pm \sqrt{4 L_{1}^{2} z_{2}^{2}\left(z_{2}^{2}+p_{22}^{2}\right)-p_{21}^{2} z_{2}^{2}}}{2 L_{1}\left(z_{2}^{2}+p_{22}^{2}\right)}
$$




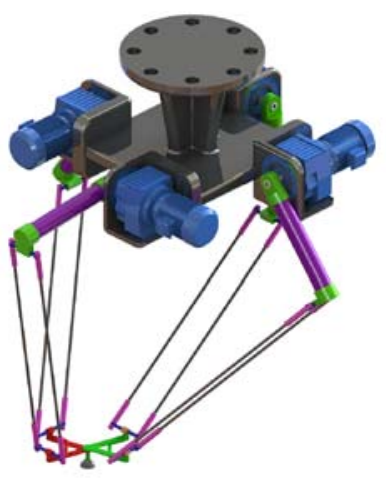

(a)

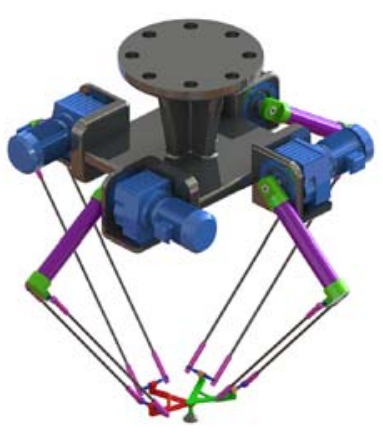

(b)
Fig. 8 Three translational motions and one rotational motion for the proposed parallel robot. (a) 3T motions; (b) 1R motion

$$
\begin{gathered}
p_{21}=L_{2}^{2}-L_{1}^{2}-x_{2}^{2}-\left(R_{1}-y_{2}\right)^{2}-z_{2}^{2}, \\
p_{22}=R_{1}-y_{2} .
\end{gathered}
$$

3) The inverse kinematic solution of Limb III is given by

$$
\begin{gathered}
\alpha_{\mathrm{III}-1}=\cos ^{-1} v_{\mathrm{III}}^{+} \quad \text { or } \quad \alpha_{\mathrm{III}-2}=2 \pi-\cos ^{-1} v_{\mathrm{III}}^{+} \\
\text {or } \alpha_{\mathrm{III}-3}=\cos ^{-1} v_{\mathrm{III}}^{-} \text {or } \quad \alpha_{\mathrm{III}-4}=2 \pi-\cos ^{-1} v_{\mathrm{III}}^{-},
\end{gathered}
$$

where

$$
\begin{gathered}
v_{\mathrm{III}}^{ \pm}=\frac{-p_{31} p_{32} \pm \sqrt{4 L_{1}^{2} z_{3}^{2}\left(z_{3}^{2}+p_{32}^{2}\right)-p_{31}^{2} z_{3}^{2}}}{2 L_{1}\left(z_{3}^{2}+p_{32}^{2}\right)}, \\
p_{31}=L_{2}^{2}-L_{1}^{2}-\left(R_{1}+x_{3}\right)^{2}-y_{3}^{2}-z_{3}^{2}, \\
p_{32}=R_{1}+x_{3} .
\end{gathered}
$$

4) The inverse kinematic solution of Limb IV is expressed as

$$
\alpha_{\mathrm{IV}-1}=\cos ^{-1} v_{\mathrm{IV}}^{+} \text {or } \alpha_{\mathrm{IV}-2}=2 \pi-\cos ^{-1} v_{\mathrm{IV}}^{+}
$$

$$
\text { or } \alpha_{\mathrm{IV}-3}=\cos ^{-1} v_{\mathrm{IV}}^{-} \text {or } \alpha_{\mathrm{IV}-4}=2 \pi-\cos ^{-1} v_{\mathrm{IV}}^{-},
$$

where

$$
\begin{gathered}
v_{\mathrm{IV}}^{ \pm}=\frac{-p_{41} p_{42} \pm \sqrt{4 L_{1}^{2} z_{4}^{2}\left(z_{4}^{2}+p_{42}^{2}\right)-p_{41}^{2} z_{4}^{2}}}{2 L_{1}\left(z_{4}^{2}+p_{42}^{2}\right)}, \\
p_{41}=L_{2}^{2}-L_{1}^{2}-x_{4}^{2}-\left(R_{1}+y_{4}\right)^{2}-z_{4}^{2} \\
p_{42}=\left(R_{1}+y_{4}\right) .
\end{gathered}
$$

By observing Eqs. (7)-(22), we can find four possible solutions for each rotational input, but only one solution is correct. Therefore, identifying the accurate solution from the possible solutions is necessary at this point. To eliminate the solution corresponding to the given configuration, two constraint conditions combined with Fig. 9 are presented below.

(1) The restricted conditions of the configuration are given by

$$
\alpha_{i}^{\min }<\alpha_{i}<\alpha_{i}^{\max }, i=1,2,3,4 .
$$

For Limb I

$$
\left\{\begin{array}{l}
\alpha_{1}^{\min }=\frac{\pi}{2}-\tan ^{-1}\left(\frac{x_{1}-R_{1}}{z}\right) \\
\alpha_{1}^{\max }=\frac{3 \pi}{2}-\tan ^{-1}\left(\frac{x_{1}-R_{1}}{z}\right)
\end{array},\right.
$$

For Limb II

$$
\left\{\begin{array}{l}
\alpha_{2}^{\min }=\frac{\pi}{2}-\tan ^{-1}\left(\frac{y_{2}-R_{1}}{z}\right) \\
\alpha_{2}^{\max }=\frac{3 \pi}{2}-\tan ^{-1}\left(\frac{y_{2}-R_{1}}{z}\right),
\end{array},\right.
$$

For Limb III

$$
\left\{\begin{array}{l}
\alpha_{3}^{\min }=\frac{\pi}{2}+\tan ^{-1}\left(\frac{x_{3}+R_{1}}{z}\right) \\
\alpha_{3}^{\max }=\frac{3 \pi}{2}+\tan ^{-1}\left(\frac{x_{3}+R_{1}}{z}\right)
\end{array},\right.
$$

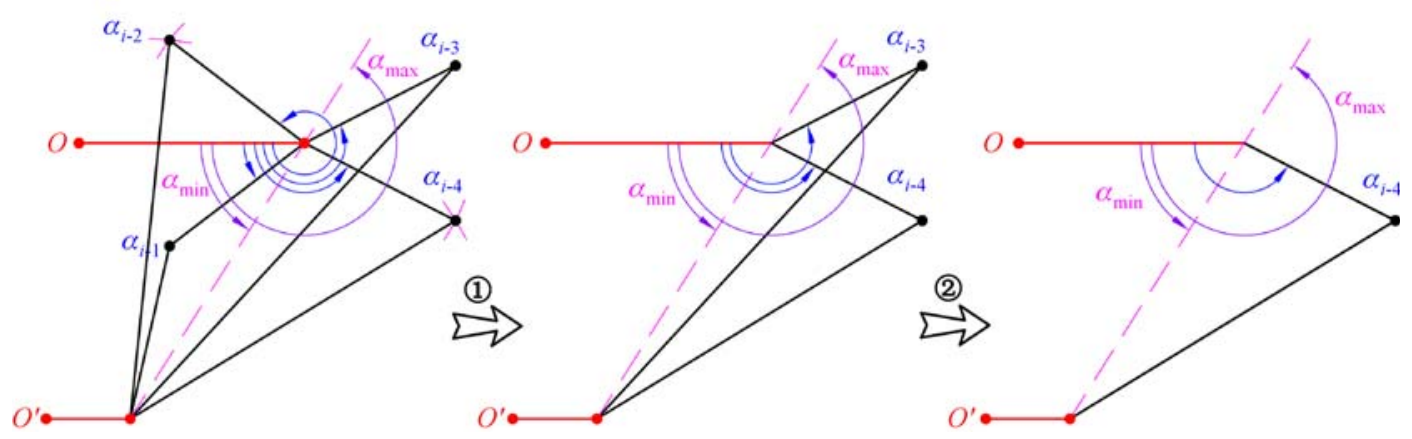

Fig. 9 Inverse solution identification process (where $i=\mathrm{I}$, II, III, and IV) 
For Limb IV

$$
\left\{\begin{array}{l}
\alpha_{4}^{\min }=\frac{\pi}{2}+\tan ^{-1}\left(\frac{y_{4}+R_{1}}{z}\right) \\
\alpha_{4}^{\max }=\frac{3 \pi}{2}+\tan ^{-1}\left(\frac{y_{4}+R_{1}}{z}\right)
\end{array} .\right.
$$

(2) The fixed length of the $B_{i} C_{i}$ bars can be solved using

$$
\left|B_{i} C_{i}\right|=L_{2}, \quad i=1,2,3,4 \text {. }
$$

According to Eqs. (23)-(28), the inverse kinematics of this mechanism can be uniquely identified.

\section{Workspace identification}

In this section, we investigate the workspace of the proposed parallel robot. The constraint conditions are both the physical dimensions and the motion/force transmission index. As shown in Fig. 10, the accelerator can be added as an attachment between the lower and upper platforms to extend the rotary output. A realization of the accelerator can be achieved by gear trains. Especially, the planetary gear trains are chosen because of their high accuracy and compact structure. The simple internal principle of a typical planetary gear train is shown in Fig. 10(b), where the axles $O_{1}$ and $O_{\mathrm{H}}$ are the input and output ends, respectively. The central Gear 3 is fixed with the lower platform, whereas the central Gear 1 is connected with the upper platform. The transmission ratio between axle $O_{1}$ and $O_{\mathrm{H}}$ can be represented by

$$
n_{1-\mathrm{H}}=1+\frac{Z_{3}}{Z_{1}}
$$

where $Z_{3}$ and $Z_{1}$ denote the tooth number of central Gears 3 and 1 , respectively. If we set 2 as the transmission ratio of the accelerator, the workspace with a rotational capability of $\pm 45^{\circ}$ should be reached, and this ability is required in most cases.

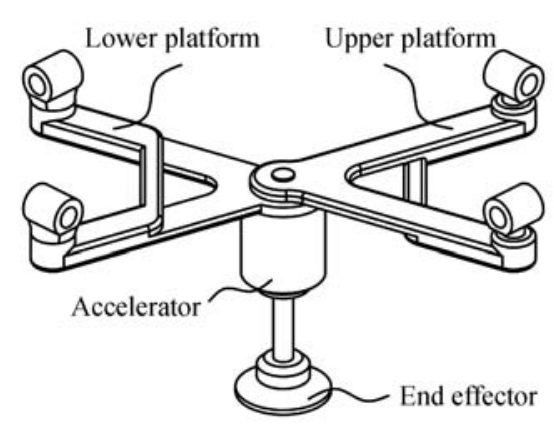

(a)

\subsection{Performance evaluation index}

The four active rotational inputs are respectively expressed as

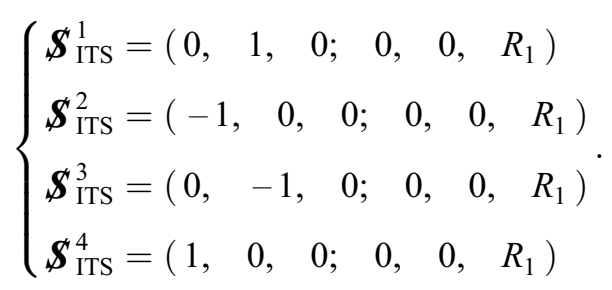

The transmission wrench screw of the $i$ th $(i=1,2,3,4)$ limb is derived using

$$
\boldsymbol{S}_{\mathrm{TWS}}^{i}=\frac{\left(B_{i} C_{i} ; \quad O B_{i} \times B_{i} C_{i}\right)}{\left|B_{i} C_{i}\right|}, i=1,2,3,4 .
$$

Assuming that the respective output twist screws of the lower and upper platforms are

$$
\begin{gathered}
\boldsymbol{S}_{\text {OTS- } l}=(\mathbf{0} ; \quad \boldsymbol{v}), \\
\boldsymbol{S}_{\text {OTS- } u}=(\boldsymbol{k} ; \quad \boldsymbol{r} \times \boldsymbol{k}+\boldsymbol{v}),
\end{gathered}
$$

where $\boldsymbol{k}$ and $\boldsymbol{r}$ are identified as

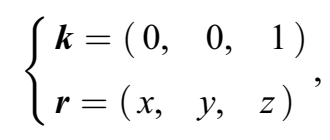

then the output twist screw of the upper platform for the $i$ th limb can be represented by $\boldsymbol{S}_{\text {OTS- } l}^{i}=\left(\mathbf{0} ; \boldsymbol{v}_{i}\right)$ and the output twist screw of the lower platform for the $i$ th limb can be represented by $\boldsymbol{S}_{\text {OTS- } u}^{i}=\left(\boldsymbol{k} ; \quad \boldsymbol{r} \times \boldsymbol{k}+\boldsymbol{v}_{i}\right)$. Considering that the power between the transmission wrench screw of Limbs II, III, and IV and the output twist screw of Limb I as a foundation, we arrive at

$$
\left\{\begin{array}{l}
\boldsymbol{S}_{\mathrm{OTS}-l}^{1} \circ \boldsymbol{S}_{\mathrm{TWS}}^{4}=0 \\
\boldsymbol{S}_{\mathrm{OTS}-u}^{1}{ }^{\circ} \boldsymbol{S}_{\mathrm{TWS}}^{2}=0 \\
\boldsymbol{S}_{\mathrm{OTS}-u}^{1} \boldsymbol{S}_{\mathrm{TWS}}^{3}=0
\end{array}\right.
$$

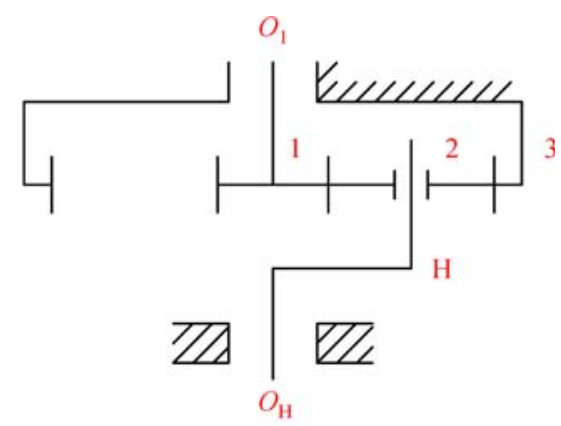

(b)

Fig. 10 The architecture of the mobile platform with accelerator. (a) Conceptual model; (b) internal principle of accelerator 
Because of the connecting of Limb I and the lower platform, the output twist screw of Limb I can be obtained by

$$
\boldsymbol{S}_{\text {OTS }}^{1}=\boldsymbol{S}_{\text {OTS- } l}^{1}=\left(\mathbf{0} ; \quad \boldsymbol{v}_{1}\right) .
$$

Similarly, the respective output twist screws of Limbs II, III, and IV are obtained using

$$
\begin{gathered}
\boldsymbol{\$}_{\mathrm{OTS}}^{2}=\boldsymbol{\$}_{\mathrm{OTS}-u}^{2}=\left(\boldsymbol{k} ; \quad \boldsymbol{r} \times \boldsymbol{k}+\boldsymbol{v}_{2}\right), \\
\boldsymbol{\$}_{\mathrm{OTS}}^{3}=\boldsymbol{\aleph}_{\mathrm{OTS}-u}^{3}=\left(\boldsymbol{k} ; \quad \boldsymbol{r} \times \boldsymbol{k}+\boldsymbol{v}_{3}\right), \\
\boldsymbol{\$}_{\mathrm{OTS}}^{4}=\boldsymbol{S}_{\text {OTS- } l}^{4}=\left(\begin{array}{ll}
\mathbf{0} & \boldsymbol{v}_{4}
\end{array}\right) .
\end{gathered}
$$

All of the $\boldsymbol{\$}_{\text {OTS }}^{2}, \boldsymbol{\$}_{\text {OTS }}^{3}$, and $\boldsymbol{S}_{\text {OTS }}^{4}$ can be respectively obtained through the equations

$$
\begin{gathered}
\left\{\begin{array}{l}
\boldsymbol{S}_{\mathrm{OTS}-u}^{2} \circ \boldsymbol{S}_{\mathrm{TWS}}^{3}=0 \\
\boldsymbol{S}_{\mathrm{OTS}-l}^{2} \circ \boldsymbol{S}_{\mathrm{TWS}}^{1}=0, \\
\boldsymbol{S}_{\mathrm{OTS}-l}^{2} \circ \boldsymbol{S}_{\mathrm{TWS}}^{4}=0
\end{array}\right. \\
\left\{\begin{array}{l}
\boldsymbol{S}_{\mathrm{OTS}-u}^{3} \circ \boldsymbol{S}_{\mathrm{TWS}}^{2}=0 \\
\boldsymbol{S}_{\mathrm{OTS}-l}^{3} \circ \boldsymbol{S}_{\mathrm{TWS}}^{1}=0, \\
\boldsymbol{S}_{\mathrm{OTS}-l}^{3} \circ \boldsymbol{S}_{\mathrm{TWS}}^{4}=0
\end{array}\right. \\
\left\{\begin{array}{l}
\boldsymbol{S}_{\mathrm{OTS}-l}^{4} \circ \boldsymbol{S}_{\mathrm{TWS}}^{1}=0 \\
\boldsymbol{S}_{\mathrm{OTS}-u}^{4}{ }^{\circ} \boldsymbol{S}_{\mathrm{TWS}}^{2}=0 . \\
\boldsymbol{S}_{\mathrm{OTS}-u}^{4} \circ \boldsymbol{S}_{\mathrm{TWS}}^{3}=0
\end{array}\right.
\end{gathered}
$$

According to the motion/force transmission index [28], an input transmission index (ITI) for the $i$ th $(i=1,2,3,4)$ limb can be derived by

$$
\xi_{i}=\frac{\left|\boldsymbol{S}_{\mathrm{ITS}}^{i}{ }^{\circ} \boldsymbol{S}_{\mathrm{TWS}}^{i}\right|}{\left|\boldsymbol{S}_{\mathrm{ITS}}^{i}{ }^{\circ} \boldsymbol{S}_{\mathrm{TWS}}^{i}\right|_{\max }},
$$

where $\boldsymbol{S}_{\mathrm{ITS}}^{i}{ }^{\circ} \boldsymbol{S}_{\mathrm{TWS}}^{i}$ is the reciprocal product between the unit input twist screw $\boldsymbol{S}_{\text {ITS }}^{i}$ and the unit transmission wrench screw $\boldsymbol{S}_{\mathrm{TWS}}^{i}, \boldsymbol{\xi}_{i}$ falls into the range $[0,1]$; in addition, the input transmission singularity occurs if $\xi_{i}=0$, whereas the mechanism is farthest away from singularity if $\xi_{i}=1$. The presented robot with parameters $R_{1}=275 \mathrm{~mm}, R_{2}=100 \mathrm{~mm}, L_{1}=365 \mathrm{~mm}$, and $L_{2}=$ $805 \mathrm{~mm}$ is taken as a presupposed condition. In the given positions $(0,0,-600 \mathrm{~mm})$, the input transmission indices of the four limbs are investigated when the rotational angle changes from $-180^{\circ}$ to $180^{\circ}$. Figure 11 shows the $\xi_{i}$ distribution. As can be seen, $\xi_{1}$ and $\xi_{4}$ are constant values, whereas $\xi_{2}$ and $\xi_{3}$ are symmetrical around $\theta=0^{\circ}$. The definite physical meaning can be described by stating that, in a fixed position, the lower platform has no translation.
This phenomenon causes the unit input twist screw $\boldsymbol{S}_{\text {ITS }}^{i}$ and unit transmission wrench screw $\boldsymbol{S}_{\text {TWS }}^{i}$ for Limbs I and IV to remain unchanged. The upper platform has a symmetrical rotation relative to $\theta=0^{\circ}$. These symmetrical relationships also apply to Limbs II and III.

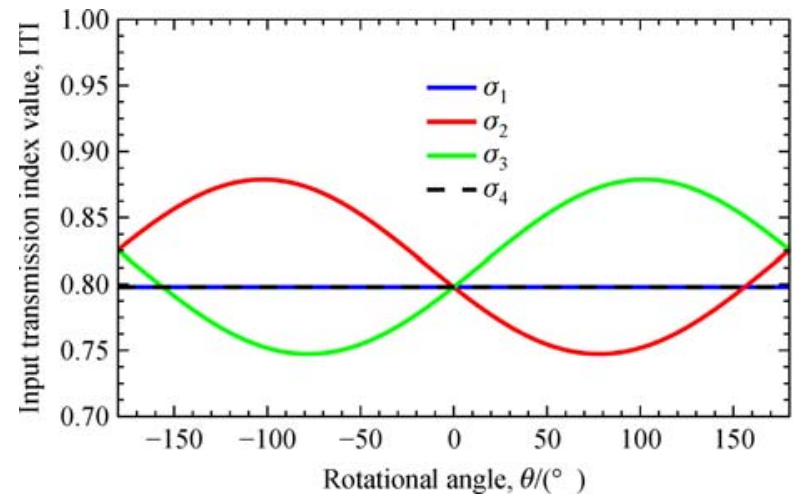

Fig. 11 Distribution of input transmission indices at point $(0,0$, $-600 \mathrm{~mm})$

Similarly, an output transmission index (OTI) for the $i$ th $(i=1,2,3,4)$ limb can be derived by

$$
\sigma_{i}=\frac{\left|\boldsymbol{S}_{\mathrm{TWS}}^{i}{ }^{\circ} \boldsymbol{S}_{\mathrm{OTS}}^{i}\right|}{\left|\boldsymbol{S}_{\mathrm{TWS}}^{i}{ }^{\circ} \boldsymbol{S}_{\mathrm{OTS}}^{i}\right|_{\max }}
$$

where $\boldsymbol{S}_{\text {TWS }}^{i}{ }^{\circ} \boldsymbol{S}_{\text {OTS }}^{i}$ is the reciprocal product between the unit transmission wrench screw $\boldsymbol{S}_{\text {TWS }}^{i}$ and the unit output twist screw $\boldsymbol{S}_{\text {OTS }}^{i}, \sigma_{i}$ falls into the range [0,1]; in addition, the output transmission singularity occurs if $\sigma_{i}=0$, whereas the mechanism is farthest away from singularity if $\sigma_{i}=1$. The output transmission singularity occurs if $\sigma_{i}=0$, whereas the mechanism is farthest away from singularity if $\sigma_{i}=1$. In the given positions $(0,0,-600$ $\mathrm{mm}$ ), the output transmission indices of the four limbs are investigated when the rotational angle changes from $-180^{\circ}$ to $180^{\circ}$. Figure 12 shows the $\sigma_{i}$ distribution. As can be seen, $\sigma_{1}$ and $\sigma_{4}$ as well as $\sigma_{2}$ and $\sigma_{3}$ are symmetrical

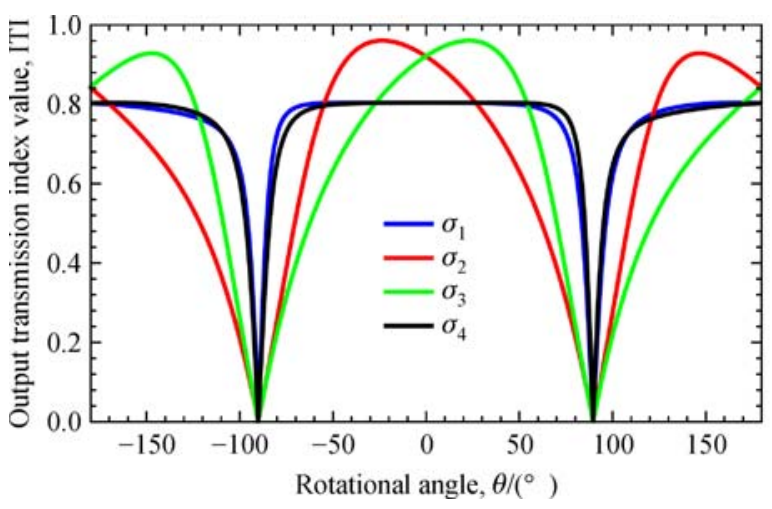

Fig. 12 Distribution of the output transmission indices at point $(0,0,-600 \mathrm{~mm})$ 
around $\theta=0^{\circ}$. The definite physical meaning can be described by stating that, for Limbs I and IV, the unit output twist screw $\boldsymbol{S}_{\text {ITS }}^{i}$ is changed symmetrically, and the unit transmission wrench screw $\boldsymbol{\$}_{\text {TWS }}^{i}$ remains unchanged. For Limbs II and III, the unit output twist screw $\boldsymbol{S}_{\text {ITS }}^{i}$ and the unit transmission wrench screw $\boldsymbol{S}_{\text {TWS }}^{i}$ are symmetrically and simultaneously change.

Two indices are defined to evaluate the rotation ability. One is the symmetrical rotational range index

$$
\theta_{\text {srr }}=\min \left\{\left|\theta_{\min }\right|, \theta_{\max }\right\},
$$

and the other is the maximum rotation range index

$$
\theta_{\mathrm{mrr}}=\theta_{\max }-\theta_{\min } \text {. }
$$

\subsection{Identification results}

By the constraint of $\sigma_{i} \geqslant 0.05$ and $\xi_{i} \geqslant 0.3$, the rotation capacity of the upper platform when $z=-600 \mathrm{~mm}$ is fully investigated. According to the physical dimensions, the

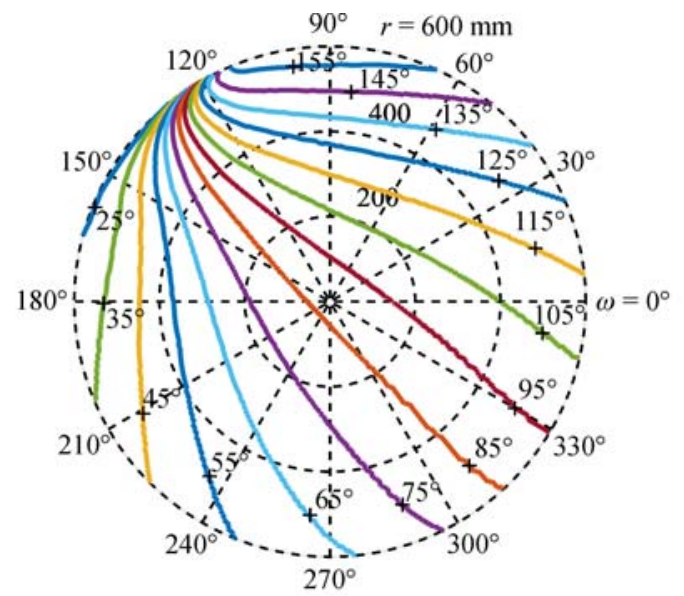

(a)

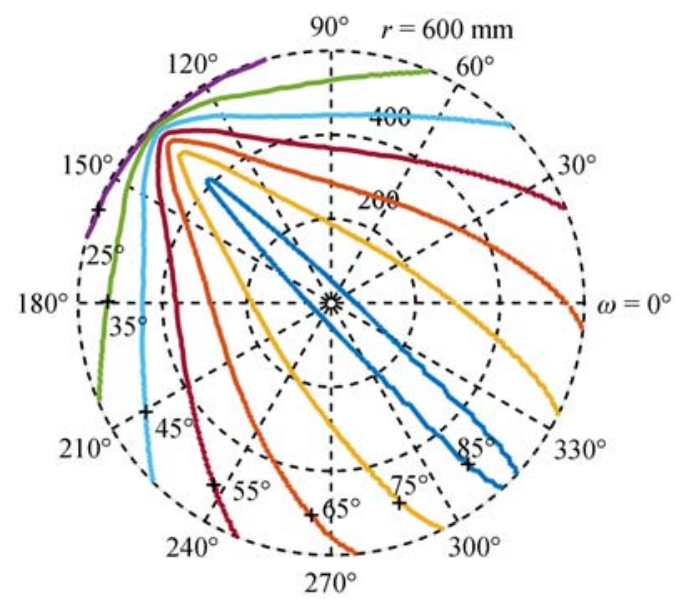

(c) size of a cross-section defined by $x=r \cos \omega, y=r \sin \omega$, where $r \in[0,600]$ (unit: $\mathrm{mm}$ ) and $\omega \in[0,2 \pi]$. The distributions of $\theta_{\max }, \theta_{\min }, \theta_{\text {srr }}$, and $\theta_{\text {mrr }}$ are plotted in Fig. 13, which clearly presents the rotation capacity. Notably, $\theta_{\text {srr }}$ and $\theta_{\text {mrr }}$ are exactly symmetrical with respect to $\omega=0.75 \pi$.

To meet the requirements of practical application, different $\theta_{\text {srr }}$ values and the corresponding atlases under different values of $z$ are shown in Fig. 14. Assuming the increasing ratio of the accelerator is 2 , the area where $\theta_{\text {srr }}$ $\geqslant 45^{\circ}$ should be focused on. Therefore, the blue segments are sub-planes of the workspace. Two types of workspaces are identified and demonstrated in the following sections.

\subsubsection{Maximum inscribed circle workspace}

The outlines of $\theta_{\text {srr }} \geqslant 45^{\circ}$ under different values of $z$ are plotted. In their intersection, a maximal inscribed circle $\phi$ : $950 \mathrm{~mm}$ can be delineated as the high rotational output area in Fig. 15. Combining the analysis results presented in Fig. 14, a maximal inscribed cylinder $\phi \times h(950 \mathrm{~mm} \times$

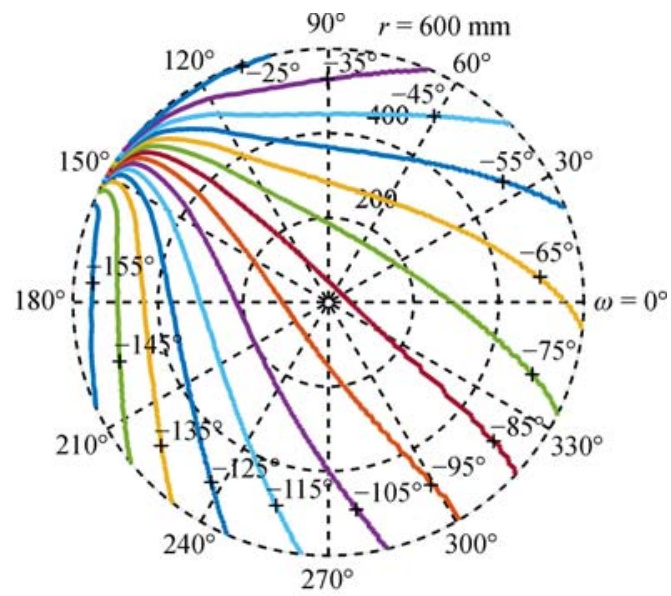

(b)

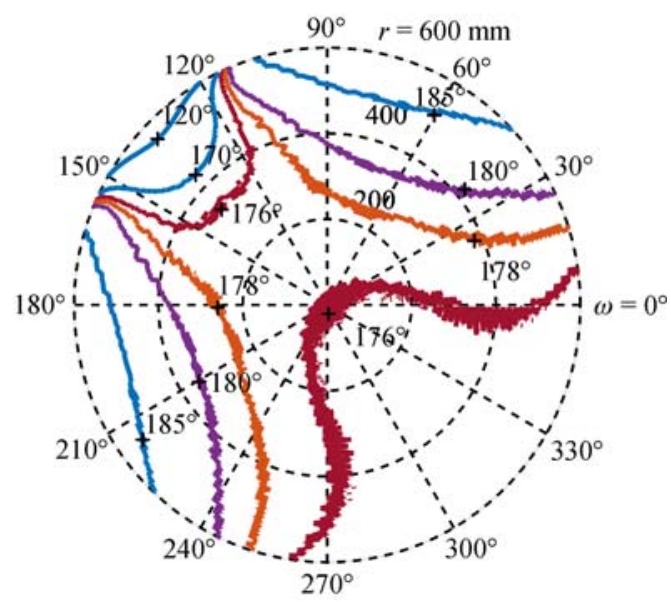

(d)

Fig. 13 Rotational capability when $z=-600 \mathrm{~mm}$. (a) Distribution of $\theta_{\max }$; (b) distribution of $\theta_{\min }$; (c) distribution of $\theta_{\text {srr }}$; (d) distribution of $\theta_{\mathrm{mrr}}$ 


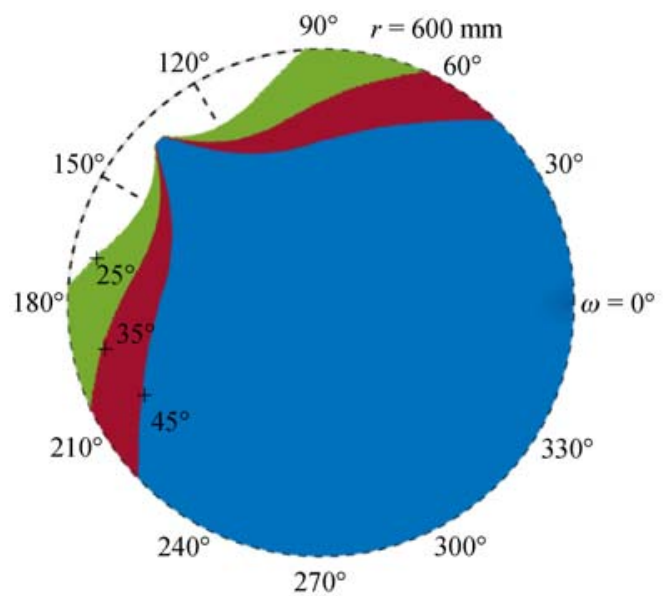

(a)

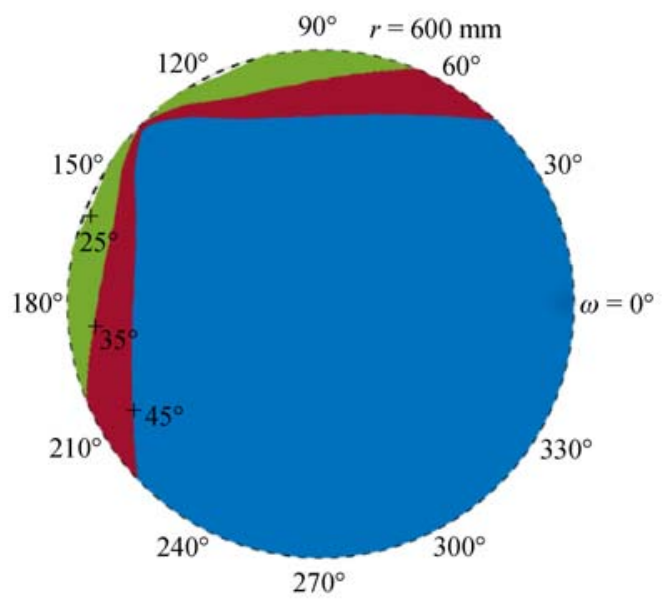

(c)

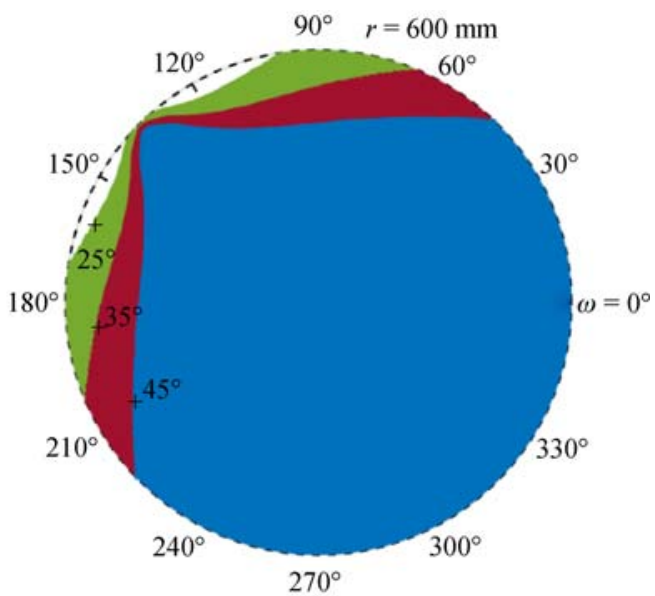

(b)

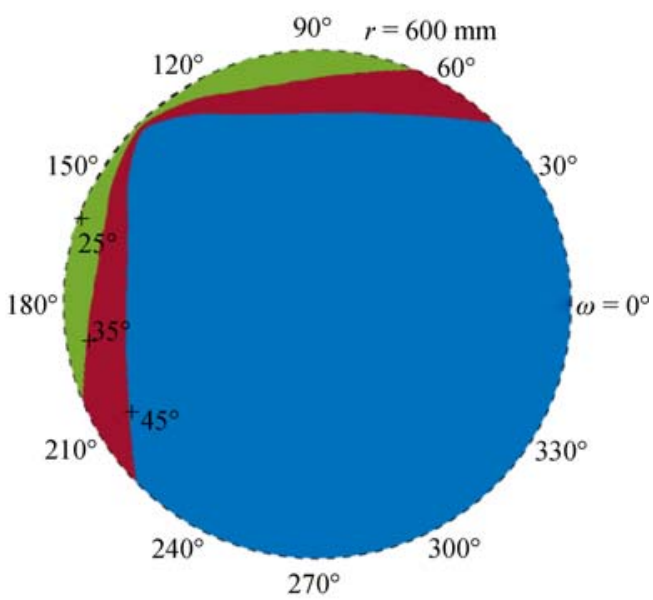

(d)

Fig. 14 Distributions of $\theta_{\text {srr }}$ under different values of $z$. (a) $z=-450 \mathrm{~mm}$; (b) $z=-500 \mathrm{~mm}$; (c) $z=-550 \mathrm{~mm}$; (d) $z=-600 \mathrm{~mm}$

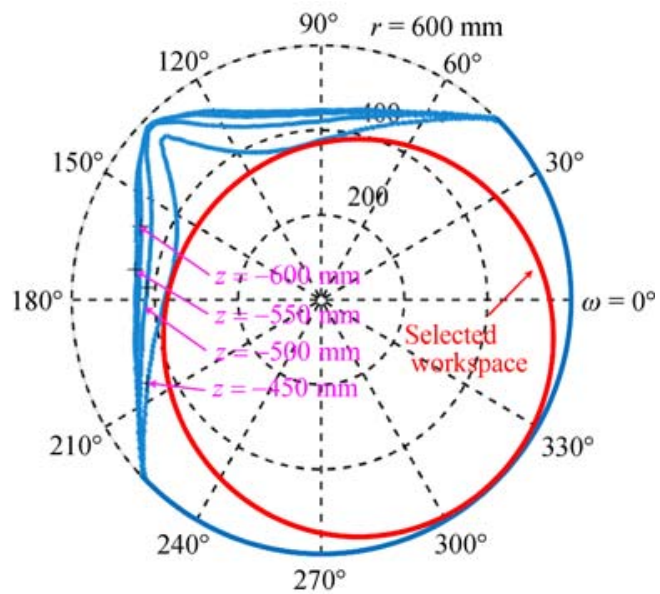

Fig. 15 Cross-sections when $z=-450,-500,-550$, and $-600 \mathrm{~mm}$

$150 \mathrm{~mm}$ ) can be identified as the workspace, which is shown in Fig. 16.
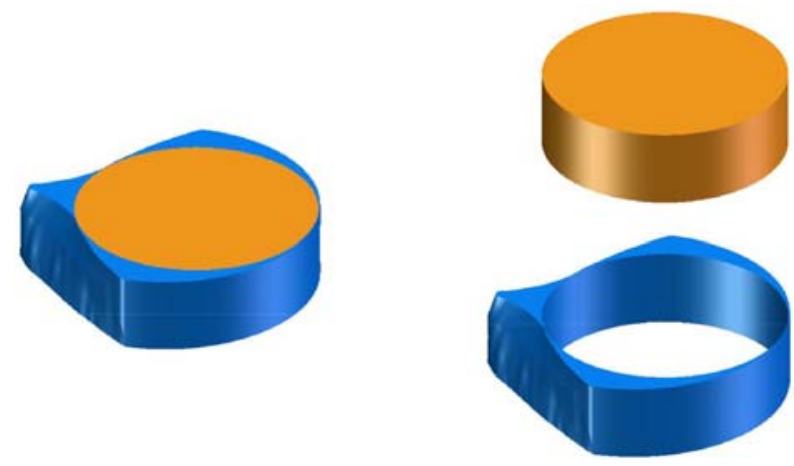

Fig. 16 The maximal inscribed circle workspace

5.2.2 Maximum symmetrical circle workspace

Considering workspace symmetry, a maximal inscribed circle $\phi: 730 \mathrm{~mm}$ can be delineated as the high rotational output area in Fig. 17. Combining the height information, a 


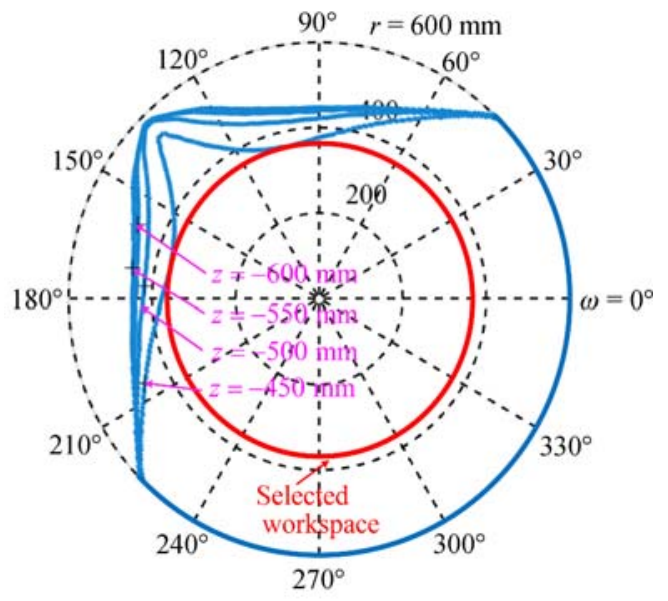

Fig. 17 Cross-sections when $z=450,-500,-550$, and $-600 \mathrm{~mm}$
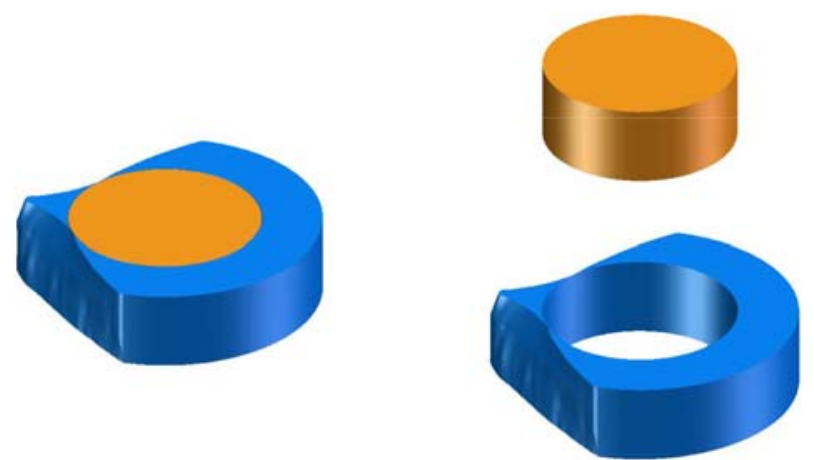

Fig. 18 The maximal symmetrical circle workspace

maximum symmetrical circle $\phi \times h(730 \mathrm{~mm} \times 150 \mathrm{~mm})$ can be delineated as the workspace, as shown in Fig. 18.

\section{Conclusions}

In this paper, the concept of a novel parallel robot for pickand-place operations with four limbs and a $1 \frac{1}{2}$ mobile platform has been proposed. Furthermore, its kinematic issues, including mobility analysis, kinematic modeling, and workspace identification have also been investigated. A line-graph method based on Grassmann line geometry was introduced to derive the freedom space, and the results indicate that the parallel robot shows the exact DOFs (3T and $1 \mathrm{R}$ ) to undertake Schönflies motion. Then, the inverse kinematics has been derived according to the principle of mechanism, and the identification conditions to pick out the practical solution have been provided. Based on the motion/force transmission index, an initial workspace far from output transmission singularity has been identified with a set of presupposed parameters. Under this precondition, the rotational capability can reach at least $\pm 45^{\circ}$ without using an additional device. Two emblematic sub-workspaces were selected as final workspace by a different definition. The analysis results presented in this paper are conducive to the parallel robot's practical application.

Acknowledgements This work was supported by the National Natural Science Foundation of China (Grant No. 51425501), and by the Beijing Municipal Science \& Technology Commission (Grant No. Z17110000 0817007). The second author wishes to acknowledge the support provided by the Alexander von Humboldt (AvH) Foundation.

Open Access This article is distributed under the terms of the Creative Commons Attribution 4.0 International License (http://creativecommons.org/ licenses/by/4.0/), which permits unrestricted use, distribution, and reproduction in any medium, provided the appropriate credit is given to the original author(s) and the source, and a link is provided to the Creative Commons license, indicating if changes were made.

\section{References}

1. Pierrot F, Company O. H4: A new family of 4-DOF parallel robots. In: Proceedings of 1999 IEEE/ASME International Conference on Advanced Intelligent Mechatronics. Atlanta: IEEE, 1999, 508-513

2. Gosselin C, Isaksson M, Marlow K, et al. Workspace and sensitivity analysis of a novel nonredundant parallel SCARA robot featuring infinite tool rotation. IEEE Robotics and Automation Letters, 2016, 1(2): 776-783

3. Urrea C, Kern J. Trajectory tracking control of a real redundant manipulator of the SCARA type. Journal of Electrical Engineering and Technology, 2016, 11(1): 215-226

4. Angeles J, Morozov A, Navarro O. A novel manipulator architecture for the production of SCARA motions. In: Proceedings of IEEE International Conference on Robotics and Automation. San Francisco: IEEE, 2000, 3: 2370-2375

5. Ancuta A, Company O, Pierrot F. Design of Lambda-Quadriglide: A new 4-DOF parallel kinematics mechanism for Schönflies motion. In: Proceedings of the ASME International Design Engineering Technical Conferences and Computers and Information in Engineering Conference. Quebec: ASME, 2010, 1131-1140

6. Li Q, Herve J M. Parallel mechanisms with bifurcation of Schöenflies motion. IEEE Transactions on Robotics, 2009, 25(1): 158-164

7. Wu G. Kinematic analysis and optimal design of a wall-mounted four-limb parallel Schönflies-motion robot for pick-and-place operations. Journal of Intelligent \& Robotic Systems, 2016, 86(34): $663-677$

8. Kim S M, Kim W, Yi B J. Kinematic analysis and optimal design of a 3T1R type parallel mechanism. In: Proceedings of IEEE International Conference on Robotics and Automation. Kobe: IEEE, 2009, 2199-2204

9. Li Q C, Xu L M, Chen Q H, et al. New family of RPR-equivalent parallel mechanisms: Design and application. Chinese Journal of Mechanical Engineering, 2017, 30(2): 217-221

10. Vischer P, Clavel R. Kinematic calibration of the parallel Delta robot. Robotica, 1998, 16(2): 207-218

11. Wahle M, Corves B. Stiffness analysis of Clavel's DELTA robot. In: 
Proceedings of International Conference on Intelligent Robotics and Applications. Berlin: Springer, 2011, 240-249

12. Clavel R. US Patent, 4976582, 1990-12-11

13. HelloTrade. ABB IRB 340 FlexPicker robots. Retrieved from http:// www.hellotrade.com/auctelia/6-new-abb-irb-340-flexpicker-robots. html, 2017-1-25

14. Krut S, Company O, Nabat V, et al. Heli4: A parallel robot for SCARA motions with a very compact traveling plate and a symmetrical design. In: Proceedings of IEEE/RSJ International Conference on Intelligent Robots and Systems. Beijing: IEEE, 2006, 1656-1661

15. Krut S, Nabat V, Company O, et al. A high-speed parallel robot for SCARA motions. In: Proceedings of IEEE International Conference on Robotics and Automation. New Orleans: IEEE, 2004, 41094115

16. Nabat V, Rodriguez M D L O, Company O, et al. Par4: Very high speed parallel robot for pick-and-place. In: Proceedings of IEEE/ RSJ International Conference on Intelligent Robots and Systems. Edmonton: IEEE, 2005, 553-558

17. Company O, Marquet F, Pierrot F. A new high-speed 4-DOF parallel robot synthesis and modeling issues. IEEE Transactions on Robotics and Automation, 2003, 19(3): 411-420

18. Pierrot F, Marquet F, Company O, et al. H4 parallel robot: Modeling, design and preliminary experiments. In: Proceedings of IEEE International Conference on Robotics and Automation. Seoul: IEEE, 2001, 3256-3261

19. Choi H B, Company O, Pierrot F, et al. Design and control of a novel 4-DOFs parallel robot H4. In: Proceedings of IEEE International Conference on Robotics and Automation. Taipei: IEEE, 2003, 1185-1190
20. Choi H B, Company O, Pierrot F, et al. Design and dynamic simulation of a novel 4-DOF parallel robot H4. Transactions of the Japan Society of Mechanical Engineers, 2004, 70(691): 798-803

21. Xie F G, Liu X J, Zhou Y H. A parallel robot with SCARA motions and its kinematic issues. In: Proceedings of the 3rd IFToMM International Symposium on Robotics and Mechatronics. Singapore: Research Publishing, 2013, 53-62

22. Xie F G, Liu X J. Design and development of a high-speed and highrotation robot with four identical arms and a single platform. Journal of Mechanisms and Robotics, 2015, 7(4): 041015

23. Xie F G, Liu X J, You Z, et al. Type synthesis of 2T1R-type parallel kinematic mechanisms and the application in manufacturing. Robotics and Computer-integrated Manufacturing, 2014, 30(1): 110

24. Yu J J, Dong X, Pei X, et al. Mobility and singularity analysis of a class of two degrees of freedom rotational parallel mechanisms using a visual graphic approach. Journal of Mechanisms and Robotics, 2012, 4(4): 041006

25. Merlet J P. Singular configurations of parallel manipulators and Grassmann geometry. International Journal of Robotics Research, 1989, 8(5): 194-212

26. Monsarrat B, Gosselin C M. Singularity analysis of a three-leg sixdegree-of-freedom parallel platform mechanism based on Grassmann line geometry. International Journal of Robotics Research, 2001, 20(4): 312-328

27. Blanding D L. Exact Constraint: Machine Design Using Kinematic Processing. New York: ASME Press, 1999

28. Liu X J, Wu C, Wang J S. A new approach for singularity analysis and closeness measurement to singularities of parallel manipulators. Journal of Mechanisms and Robotics, 2012, 4(4): 041001 